\title{
SCOTT'S FORMULA AND HURWITZ GROUPS
}

\author{
M.A. PELLEGRINI AND M.C. TAMBURINI BELLANI
}

\begin{abstract}
This paper continues previous work, based on systematic use of a formula of L. Scott, to detect Hurwitz groups. It closes the problem of determining the finite simple groups contained in $\mathrm{PGL}_{n}(\mathbb{F})$ for $n \leq 7$ which are Hurwitz, where $\mathbb{F}$ is an algebraically closed field. For the groups $G_{2}(q)$, $q \geq 5$, and the Janko groups $J_{1}$ and $J_{2}$ it provides explicit $(2,3,7)$-generators.
\end{abstract}

\section{INTRODUCTION}

Let $\mathbb{F}$ be an algebraically closed field of any characteristic. Consider a finitely generated group $H$ and a representation $\Phi: H \rightarrow \mathrm{GL}(V)$, with representation space $V \cong \mathbb{F}^{m}$. For any subset $A$ of $H$ define:

$$
\begin{aligned}
& d_{V}^{A}=\operatorname{dim}\{v \in V \mid \Phi(a) v=v, \text { for all } a \in A\}, \\
& \hat{d}_{V}^{A}=\operatorname{dim}\left\{v \in V \mid \Phi(a)^{T} v=v, \text { for all } a \in A\right\} .
\end{aligned}
$$

Moreover, for $A=\{a\}$, set $d_{V}^{A}=d_{V}^{a}$. By a special case of the well known formula of L. Scott [17] (see also [18]), if $H=\langle x, y\rangle$, then

$$
d_{V}^{x}+d_{V}^{y}+d_{V}^{x y} \leq m+d_{V}^{H}+\hat{d}_{V}^{H} .
$$

This formula has a crucial impact on Hurwitz generation in low rank, as already suggested by Scott himself, and made more evident by the work of A. Zalesski 7, 24 and others after him.

To illustrate this claim, it is convenient to introduce some terminology. A triple $(x, y, x y)$ in $\mathrm{GL}_{n}(\mathbb{F})^{3}$ is called irreducible whenever $\langle x, y\rangle$ is an irreducible subgroup of $\mathrm{GL}_{n}(\mathbb{F})$. It is called rigid when it is irreducible and equality holds in (11), with respect to the conjugation action $\Phi$ of $H=\langle x, y\rangle$ on $V=\operatorname{Mat}_{n}(\mathbb{F})$. Finally $(x, y, x y)$ is said to be a $(2,3,7)$-triple whenever the projective images of $x, y, x y$ have respective orders $2,3,7$. Clearly all these properties are preserved by conjugation.

In [19] were classified (up to multiplication by scalars) the admissible similarity invariants, equivalently the admissible Jordan canonical forms (e.g., see [9, 3.10]), of $x, y, x y$ in an irreducible $(2,3,7)$-triple $(x, y, x y) \in \mathrm{GL}_{n}(\mathbb{F})^{3}, n \leq 7$. It turns out that such triples are all rigid if and only if $n \leq 5$. For $n=6,7$, the similarity invariants of the non-rigid ones (parametrized in [25] and [20]) are respectively:

$$
t^{2}+1, t^{2}+1, t^{2}+1 ; \quad t^{3}-1, t^{3}-1 ; \quad t^{6}+t^{5}+t^{4}+t^{3}+t^{2}+t+1,
$$

for $n=6$ (see [19, 1.1.1 page 349 and 2.1.1 page 350]), and

$$
t+1, t^{2}-1, t^{2}-1, t^{2}-1 ; \quad t-1, t^{3}-1, t^{3}-1 ; \quad t^{7}-1,
$$

for $n=7$ (see [19, 1.1 page 351]).

2010 Mathematics Subject Classification. 20G40, $20 \mathrm{~F} 05$.

Key words and phrases. Hurwitz generations; trilinear forms; $G_{2}(q)$. 
An irreducible triple of type (2) generates a symplectic group. The irreducibility of a triple of type (3) forces char $\mathbb{F} \neq 2$ and the corresponding group is orthogonal. The proof of these facts uses techniques based on Scott's formula (see Lemma 2.1).

In this paper we continue the analysis started in [19] and [20] with respect to the non-rigid triples. In particular we prove the following two results:

Theorem 1.1. Suppose that $\mathbb{F}$ is an algebraically closed field. Let $H_{7}$ be an irreducible subgroup of $\mathrm{SL}_{7}(\mathbb{F})$ generated by a non-rigid $(2,3,7)$-triple, i.e. a triple with similarity invariants (3). Then char $\mathbb{F} \neq 2$ and $H_{7}$ is a subgroup of $G_{2}(\mathbb{F})$.

Theorem 1.2. Suppose that $\mathbb{F}$ is an algebraically closed field of characteristic 2. Let $H_{6}$ be an irreducible subgroup of $\mathrm{SL}_{6}(\mathbb{F})$ generated by a non-rigid $(2,3,7)$-triple, i.e., a triple with similarity invariants (2). Then $H_{6}$ is a subgroup of $G_{2}(\mathbb{F})$.

These two theorems allow to complete the classification of the finite simple groups, admitting absolutely irreducible projective representations of degree $n \leq 7$, which are Hurwitz. Among such groups, those which are generated by rigid triples, in particular those which admit irreducible projective representations of degree $n \leq 5$, have already been classified (see 19]). Thus we are left with those generated by irreducible non-rigid triples. For $n=6$ and char $\mathbb{F} \neq 2$, by Lemma2.1(i) they are contained in $\operatorname{Sp}_{6}(q), q$ odd, where $q$ stands for the order of a finite field. Otherwise, they are contained in $G_{2}(q)$ by Theorems 1.1 and 1.2 Thus the classification can be completed analyzing $\operatorname{Sp}_{6}(q), G_{2}(q)$ and their subgroups.

The group $\operatorname{PSp}_{6}(q)$ is not Hurwitz for $q=3$ and for $q$ even (see 24]; for $q$ even this fact also follows from Theorem 1.2). Hurwitz generators of $\operatorname{PSp}_{6}(q), q \geq 5$ odd, are given in 22 . On the other hand, by an old, non-constructive result of G. Malle, $G_{2}(q)$ itself is a Hurwitz group if, and only if, $q \geq 5$. Explicit $(2,3,7)$-generators for the Ree groups ${ }^{2} G_{2}\left(3^{2 a+1}\right)$ can be found in [23].

For the reader's convenience we state the full classification. Given an integer $u$ and a prime $p$, coprime to $u$, we denote by $o_{u}(p)$ the order of $p(\bmod u)$, i.e. the order of $p+u \mathbb{Z}$ seen as an element of the group $(\mathbb{Z} / u \mathbb{Z})^{*}$.

Theorem 1.3. Let $G$ be a finite simple group admitting an absolutely irreducible projective representation of degree $n \leq 7$. Then $G$ is Hurwitz if, and only if, it is isomorphic to one of the following:

(i) $\mathrm{PSL}_{2}(p)$ when $p \equiv 0, \pm 1(\bmod 7), \mathrm{PSL}_{2}\left(p^{3}\right)$ when $p \equiv \pm 2, \pm 3(\bmod 7)$ 13 ;

(ii) $\operatorname{PSL}_{5}\left(p^{n_{5}}\right)$ when $p \neq 5,7$ and $n_{5}$ is odd, $\operatorname{PSU}_{5}\left(7^{4}\right)$ and $\operatorname{PSU}_{5}\left(p^{n_{5}}\right)$ when $p \neq 5,7$ and $n_{5}$ is even, where $n_{5}$ is $\mathrm{O}_{5}(p) \cdot \mathrm{O}_{7}\left(p^{2}\right)$ [21;

(iii) $\operatorname{PSL}_{6}\left(p^{n_{6}}\right)$ when $p \neq 3$ and $n_{6}$ is odd, $\mathrm{PSU}_{6}\left(p^{n_{6}}\right)$ when $p \neq 3$ and $n_{6}$ is even, where $n_{6}=\mathrm{o}_{9}(p)$ [19];

(iv) $\mathrm{PSL}_{7}\left(p^{n_{7}}\right)$ when $p \neq 7$ and $n_{7}$ is odd, $\operatorname{PSU}_{7}\left(p^{n_{7}}\right)$ when $p \neq 7$ and $n_{7}$ is even, where $n_{7}=\mathrm{o}_{49}(p)[19$;

(v) $\operatorname{PSp}_{6}(q)$ when $q \geq 5$ is odd 22 ;

(vi) $G_{2}(q)$ when $q \geq 5$ and ${ }^{2} G_{2}\left(3^{2 a+1}\right)$ for all $a \geq 1$ [14;

(vii) $J_{1}\left[16, J_{2}[8\right.$.

The groups in items (i)-(iv) of previous theorem are all generated by rigid triples. Clearly rigidity restricts the isomorphism types: at most one for fixed $p$ and $n$. For an interesting discussion of this aspect we refer to [15].

In the last Section we provide explicit Hurwitz generators of $G_{2}(q), q \geq 5$, and of the Janko groups $J_{1}$ and $J_{2}$, respectively over $\mathbb{F}_{11}$ and $\mathbb{F}_{4}$. 
Acknowledgments. We are indebted to Maxim Vsemirnov for useful suggestions and discussions.

\section{Preliminary Results}

Let $\left(x_{n}, y_{n}, x_{n} y_{n}\right)$ be an irreducible triple in $\mathrm{GL}_{n}(\mathbb{F})^{3}$, where $\mathbb{F}$ is an algebraically closed field, and set $H_{n}=\left\langle x_{n}, y_{n}\right\rangle$. We consider the diagonal action of $\mathrm{GL}_{n}(\mathbb{F})$ on the space $\mathbb{F}^{n} \otimes \mathbb{F}^{n}$, identified with $\operatorname{Mat}_{n}(\mathbb{F})$, namely the action $B \mapsto g B g^{T}$ for all $B \in \operatorname{Mat}_{n}(\mathbb{F}), g \in \mathrm{GL}_{n}(\mathbb{F})$. The symmetric square $S=S\left(\mathbb{F}^{n}\right)$ and the exterior square power $\Lambda^{2}=\Lambda^{2}\left(\mathbb{F}^{n}\right)$ can be identified, respectively, with the spaces of symmetric matrices and antisymmetric matrices with zero-diagonal. The spaces $S$ and $\Lambda^{2}$, being $\mathrm{GL}_{n}(\mathbb{F})$-invariant, give rise to representations of $H_{n}$ on $\mathbb{F}$ of respective degrees $\frac{n(n+1)}{2}$ and $\frac{n(n-1)}{2}$. By [19, Lemma 1(i)], in the first representation one has

$$
\hat{d}_{S}^{H_{n}} \leq d_{S}^{H_{n}} \leq 1 .
$$

Here the notation is the same defined at the beginning of the Introduction.

Lemma 2.1. In the above notation, the following facts hold:

(i) If $n=6$ and $\left(x_{6}, y_{6}, x_{6} y_{6}\right)$ is an irreducible triple in $\mathrm{GL}_{6}(\mathbb{F})^{3}$ with similarity invariants (2), then $H_{6}=\left\langle x_{6}, y_{6}\right\rangle$ is contained in $\mathrm{Sp}_{6}(\mathbb{F})$.

(ii) If $n=7$ and $\left(x_{7}, y_{7}, x_{7} y_{7}\right)$ is an irreducible triple in $\mathrm{GL}_{7}(\mathbb{F})^{3}$ with similarity invariants (3), then char $\mathbb{F} \neq 2$ and $H_{7}=\left\langle x_{7}, y_{7}\right\rangle$ is contained in $\Omega_{7}(\mathbb{F})$.

Proof. (i) If char $\mathbb{F} \neq 2$ we consider the action of $H_{6}$ on $\Lambda^{2}=\Lambda^{2}\left(\mathbb{F}^{6}\right)$, as in 25]. Then $\mathbb{F}^{6} \otimes \mathbb{F}^{6}=S \oplus \Lambda^{2}$, whence $\hat{d}_{\Lambda^{2}}^{H_{6}}=d_{\Lambda^{2}}^{H_{6}^{T}}$. From $d_{\Lambda^{2}}^{x_{6}}=9, d_{\Lambda^{2}}^{y_{6}} \geq 5, d_{\Lambda^{2}}^{x_{6} y_{6}} \geq 3$ and $\operatorname{dim} \Lambda^{2}=15$, we get $d_{\Lambda^{2}}^{H_{6}}+\hat{d}_{\Lambda^{2}}^{H_{6}} \geq 2$. Since $d_{\Lambda^{2}}^{H_{6}} \leq 1$ and $d_{\Lambda^{2}}^{H_{6}^{T}} \leq 1$, by the irreducibility of $H_{6}$, it follows $d_{\Lambda^{2}}^{H_{6}}=d_{\Lambda^{2}}^{H_{6}^{T}}=1$. Hence $H_{6}$ fixes a non-zero alternating antisymmetric form $J$, which is non-degenerate by the irreducibility of $H_{6}$. We conclude $H_{6} \leq \operatorname{Sp}_{6}(\mathbb{F})$.

Now assume char $\mathbb{F}=2$. Then $d_{S}^{x_{6}}=12, d_{S}^{y_{6}}=7$ and $d_{S}^{x_{6} y_{6}}=3$. Since $\operatorname{dim} S=$ 21, from (41) and Scott's formula (11) it follows either $d_{S}^{H_{6}}=\hat{d}_{S}^{H_{6}}=1$ or $d_{S}^{H_{6}}=1$ and $\hat{d}_{S}^{H_{6}}=0$. In the first case $H_{6} \leq \Omega_{6}(\mathbb{F}) \leq \operatorname{Sp}_{6}(\mathbb{F})$, in the second $H_{6} \leq \operatorname{Sp}_{6}(\mathbb{F})$ by [19, Lemma 1 (ii)-(iii)].

(ii) We have $d_{S}^{x_{7}}=16, d_{S}^{y_{7}}=10, d_{S}^{x_{7} y_{7}}=4$. Since $\operatorname{dim} S=28$, from (11) we get $d_{S}^{H_{7}}+\hat{d}_{S}^{H_{7}} \geq 2$. Again [19, Lemma 1] gives char $\mathbb{F} \neq 2, d_{S}^{H_{7}}=\hat{d}_{S}^{H_{7}}=1$, and $H_{7}$ orthogonal. Since $H_{7}$ is $(2,3,7)$-generated, it coincides with its derived subgroup and so it is contained in $\Omega_{7}(\mathbb{F})$.

When $\operatorname{char} \mathbb{F}=2, \operatorname{Sp}_{6}(\mathbb{F})$ is isomorphic to the orthogonal group $\Omega_{7}(\mathbb{F})$, by a classical result of Dieudonné [6]. Indeed, call $Q$ the quadratic form which defines $\Omega_{7}(\mathbb{F})$, call $g$ the associated symmetric bilinear form of rank 6 and $\left\langle v_{0}\right\rangle$ the radical of $g$. Then $\Omega_{7}(\mathbb{F})$ fixes $\left\langle v_{0}\right\rangle$ and induces a symplectic group on $\frac{\mathbb{F}^{7}}{\left\langle v_{0}\right\rangle}$. Since every symplectic transformation has determinant $1, \Omega_{7}(\mathbb{F})$ fixes $v_{0}$. Hence, with respect to any basis of $\mathbb{F}^{7}$ having $v_{0}$ as first vector, $\Omega_{7}(\mathbb{F})$ consists of matrices

$$
h=\left(\begin{array}{cc}
1 & a_{h}^{T} \\
0 & h_{6}
\end{array}\right), a_{h} \in \mathbb{F}^{6}, h_{6} \in \operatorname{Sp}_{6}(\mathbb{F}) .
$$

The map $h \mapsto h_{6}$ is an isomorphism. We use its inverse $h_{6} \mapsto h$. The image $\left(\tilde{x}_{7}, \tilde{y}_{7}, \tilde{x}_{7} \tilde{y}_{7}\right)$ of $\left(x_{6}, y_{6}, x_{6} y_{6}\right)$ in $\Omega_{7}(\mathbb{F})$ has similarity invariants (3). Since 
the eigenspace of $\tilde{x}_{7} \tilde{y}_{7}$ relative to 1 has dimension 1 , it must coincide with $\left\langle v_{0}\right\rangle$. Let $\mathcal{B}=\left\{v_{0}, v_{1}, v_{2}, v_{-3}, v_{-1}, v_{-2}, v_{3}\right\}$ be a basis of eigenvectors of $\tilde{x}_{7} \tilde{y}_{7}$, with $\left(\tilde{x}_{7} \tilde{y}_{7}\right) v_{i}=\varepsilon^{-i} v_{i}$ for $i=0, \pm 1, \pm 2, \pm 3$, where $\varepsilon \in \mathbb{F}$ is a primitive 7 -th root of 1. With respect to $\mathcal{B}$ the Gram matrix $J$ of the bilinear form $g$ fixed by $\left\langle\tilde{x}_{7}, \tilde{y}_{7}\right\rangle$ is $J=\left(\begin{array}{cc}0 & 0 \\ 0 & J_{6}\end{array}\right)$ with $J_{6}$ fixed by $\left\langle x_{6}, y_{6}\right\rangle$. Multiplying $v_{1}, v_{2}, v_{-3}$ by scalar multiples, if necessary, we have $J_{6}=\left(\begin{array}{cc}0 & I_{3} \\ I_{3} & 0\end{array}\right)$. Hence we may suppose:

$$
\tilde{x}_{7}=\left(\begin{array}{cc}
1 & a_{x}^{T} \\
0 & x_{6}
\end{array}\right), \quad \tilde{y}_{7}=\left(\begin{array}{cc}
1 & a_{x}^{T} x_{6} y_{6} \\
0 & y_{6}
\end{array}\right), \quad \tilde{x}_{7} \tilde{y}_{7}=\left(\begin{array}{cc}
1 & 0 \\
0 & x_{6} y_{6}
\end{array}\right) .
$$

The conditions $\tilde{x}_{7}^{2}=I, J_{6} x_{6}=x_{6}^{T} J_{6}$ and the choice of $\mathcal{B}$ give $a_{x}^{T} x_{6}=a_{x}^{T}$ and

$$
\begin{gathered}
x_{6}=\left(\begin{array}{cc}
A & B \\
C & A^{T}
\end{array}\right), B=B^{T}, C=C^{T}, \\
x_{6} y_{6}=\operatorname{diag}\left(\varepsilon^{-1}, \varepsilon^{-2}, \varepsilon^{3}, \varepsilon^{1}, \varepsilon^{2}, \varepsilon^{-3}\right) .
\end{gathered}
$$

\section{Proofs of Theorems 1.1 and 1.2}

The group $G_{2}(\mathbb{F})$ can be defined as the subgroup of $\mathrm{GL}_{7}(\mathbb{F})$ fixing a particular alternating trilinear form, called the Dickson form (see [1]). For the reader's convenience, we recall some basic facts and definitions concerning alternating $m$-linear forms on $V=\mathbb{F}^{n}(n \geq m)$.

An $m$-linear map $f: V^{m} \rightarrow \mathbb{F}$ is said to be alternating if $f\left(v_{1}, \ldots, v_{m}\right)=0$ whenever $v_{i}=v_{j}$ for some $i \neq j$. We define

$$
\wedge^{m}(V)=\frac{\otimes_{m} V}{\left\langle v_{1} \otimes \ldots \otimes v_{m}: v_{i}=v_{j} \text { for some } i \neq j\right\rangle} .
$$

By $v_{1} \wedge \ldots \wedge v_{m}$ we denote the image of $v_{1} \otimes \ldots \otimes v_{m}$ under the canonical projection of $\otimes_{m} V$ onto $\wedge^{m}(V)$. The spaces $\wedge^{m}(V)$ and $\wedge^{m}\left(V^{*}\right)$, where $V^{*}$ denotes the dual of $V$, are dual to each other, under the bilinear map

$$
\left(v_{1}^{*} \wedge \ldots \wedge v_{m}^{*}, u_{1} \wedge \ldots \wedge u_{m}\right) \mapsto \operatorname{det}\left(v_{k}^{T} u_{j}\right)
$$

(e.g., see [12, Chapter XIX]). Hence, the space of alternating $m$-linear forms on $V$ can be identified with the skew-symmetric tensor product $\wedge^{m}\left(V^{*}\right)$ acting on $V^{m}$ via:

$$
\left(v_{1}^{*} \wedge \ldots \wedge v_{m}^{*}\right)\left(u_{1}, \ldots, u_{m}\right)=\operatorname{det}\left(v_{k}^{T} u_{j}\right) .
$$

We may define a representation $\Phi: \mathrm{GL}(V) \rightarrow \mathrm{GL}\left(\wedge^{m}\left(V^{*}\right)\right)$ by setting:

$$
\left[\Phi(g)\left(v_{1}^{*} \wedge \ldots \wedge v_{m}^{*}\right)\right]\left(u_{1}, \ldots, u_{m}\right)=\left(v_{1}^{*} \wedge \ldots \wedge v_{m}^{*}\right)\left(g^{-1} u_{1}, \ldots, g^{-1} u_{m}\right),
$$

namely

$$
\Phi(g)\left(v_{1}^{*} \wedge \ldots \wedge v_{m}^{*}\right)=\left(g^{-T} v_{1}\right)^{*} \wedge \ldots \wedge\left(g^{-T} v_{m}\right)^{*} .
$$

We claim that $\Phi\left(g^{T}\right)=\Phi(g)^{T}$ for all $g$. In particular, the representation $g \mapsto$ $\Phi\left(g^{-T}\right)$ is the dual of $\Phi$. Indeed, let $\left\{e_{1}, \ldots, e_{n}\right\}$ and $\left\{e_{1}^{*}, \ldots, e_{n}^{*}\right\}$ denote respective bases for $V$ and for the dual $V^{*}$ of $V$. Setting

$$
\mathcal{B}=\left\{e_{i_{1}}^{*} \wedge \ldots \wedge e_{i_{m}}^{*} \mid 1 \leq i_{1}<i_{2}<\ldots<i_{m} \leq n\right\}
$$

we obtain a basis of $\wedge^{m}\left(V^{*}\right)$. By the above

$$
\Phi(g)\left(e_{i_{1}}^{*} \wedge \ldots \wedge e_{i_{m}}^{*}\right)=\left(g^{-T} e_{i_{1}}\right)^{*} \wedge \ldots \wedge\left(g^{-T} e_{i_{m}}\right)^{*}
$$




$$
=\sum_{1 \leq k_{1} \leq \cdots \leq k_{m} \leq n} \operatorname{det}\left(\gamma_{i_{1}, \ldots, i_{m}}^{k_{1}, \ldots, k_{m}}\right)\left(e_{k_{1}}^{*} \wedge \ldots \wedge e_{k_{m}}^{*}\right)
$$

where $\gamma_{i_{1}, \ldots, i_{m}}^{k_{1}, \ldots, k_{m}}$ is the submatrix obtained from $g^{-T}$ considering rows $i_{1}, \ldots, i_{m}$ and columns $k_{1}, \ldots, k_{m}$. The same calculation leads to

$$
\Phi\left(g^{T}\right)\left(e_{i_{1}}^{*} \wedge \ldots \wedge e_{i_{m}}^{*}\right)=\sum_{1 \leq k_{1} \leq \cdots \leq k_{m} \leq n} \operatorname{det}\left(\gamma_{k_{1}, \ldots, k_{m}}^{i_{1}, \ldots, i_{m}}\right)\left(e_{k_{1}}^{*} \wedge \ldots \wedge e_{k_{m}}^{*}\right)
$$

Identifying $\Phi(g)$ and $\Phi\left(g^{T}\right)$ with their matrices with respect to $\mathcal{B}$ we have $\Phi\left(g^{T}\right)=$ $\Phi(g)^{T}$, whence our claim.

When $m=3$ and $n=7$ we may take a basis $\left\{v_{0}, v_{1}, v_{2}, v_{3}, v_{-1}, v_{-2}, v_{-3}\right\}$ and consider the following alternating trilinear form, called a Dickson form:

$$
v_{0}^{*} \wedge v_{1}^{*} \wedge v_{-1}^{*}+v_{0}^{*} \wedge v_{2}^{*} \wedge v_{-2}^{*}+v_{0}^{*} \wedge v_{3}^{*} \wedge v_{-3}^{*}+v_{1}^{*} \wedge v_{2}^{*} \wedge v_{-3}^{*}+v_{-1}^{*} \wedge v_{-2}^{*} \wedge v_{3}^{*} .
$$

Our proofs of Theorems 1.1 and 1.2 are based on key results of Aschbacher [1, Theorem 5]. The case $n=7$ turns out to be much easier to handle than the case $n=6$. For this reason we consider it firstly.

Proof of Theorem 1.1. Let $H_{7}$ be an irreducible subgroup of $\mathrm{SL}_{7}(\mathbb{F})$, generated by a non rigid $(2,3,7)$-triple $\left(x_{7}, y_{7}, x_{7} y_{7}\right)$. Its similarity invariants are those in (3) by [20]. Moreover char $\mathbb{F} \neq 2$ and $H_{7} \leq \Omega_{7}(\mathbb{F})$ (see Lemma 2.1). Consider the space $V=\mathbb{F}^{7}$ with basis $\left\{e_{1}, \ldots, e_{7}\right\}$. As done before, we may identify the space of the alternating trilinear forms defined on $V$ with the space $\wedge^{3}\left(V^{*}\right)$ and consider the representation $\Phi: \mathrm{GL}(V) \rightarrow \mathrm{GL}\left(\wedge^{3}\left(V^{*}\right)\right)$ previously introduced. We apply formula (11) to this action:

$$
d_{\Lambda^{3}\left(V^{*}\right)}^{x_{7}}+d_{\Lambda^{3}\left(V^{*}\right)}^{y_{7}}+d_{\Lambda^{3}\left(V^{*}\right)}^{x_{7} y_{7}} \leq 35+d_{\Lambda^{3}\left(V^{*}\right)}^{H_{7}}+\hat{d}_{\Lambda^{3}\left(V^{*}\right)}^{H_{7}} .
$$

Considering the canonical forms of $x_{7}, y_{7}$ and $x_{7} y_{7}$ we obtain $d_{\Lambda^{3}\left(V^{*}\right)}^{x_{7}}=19$, $d_{\Lambda^{3}\left(V^{*}\right)}^{y_{7}}=13$ and $d_{\Lambda^{3}\left(V^{*}\right)}^{x_{7} y_{7}}=5$, whence

$$
d_{\Lambda^{3}\left(V^{*}\right)}^{H_{7}}+\hat{d}_{\Lambda^{3}\left(V^{*}\right)}^{H_{7}} \geq 2 .
$$

As $\Phi(h)^{T}=\Phi\left(h^{T}\right)$ for all $h \in H_{7}$ we obtain that $\hat{d}_{\Lambda^{3}\left(V^{*}\right)}^{H_{7}}=d_{\Lambda^{3}\left(V^{*}\right)}^{H^{T}}$. Further, since $H_{7}$ and $H_{7}^{T}$ are absolutely irreducible, by [1, Theorem 5(2)],

$$
0 \leq d_{\Lambda^{3}\left(V^{*}\right)}^{H_{7}} \leq 1, \quad 0 \leq d_{\Lambda^{3}\left(V^{*}\right)}^{H_{T}^{T}} \leq 1
$$

It follows that $d_{\Lambda^{3}\left(V^{*}\right)}^{H_{7}}=\hat{d}_{\Lambda^{3}\left(V^{*}\right)}^{H_{7}}=1$. Thus $H_{7}$ fixes a non-zero alternating trilinear form which, by [1, Theorem 5(5)], must be a Dickson form. We conclude that $H_{7} \leq G_{2}(\mathbb{F})$.

We now turn to the proof of Theorem 1.2 and so, from now on, we assume char $\mathbb{F}=2$ and keep notation (6). A fundamental step of the proof is that, whenever $H_{6}=\left\langle x_{6}, y_{6}\right\rangle$ is absolutely irreducible, then $H_{7}=\left\langle\tilde{x}_{7}, \tilde{y}_{7}\right\rangle$ fixes a non-zero alternating trilinear form. This will be done in Proposition 3.5] that requires some preliminary facts established in Proposition 3.1] and Lemmas 3.3 and 3.4 .

Proposition 3.1. Let $K$ be an irreducible subgroup of $\mathrm{SL}_{6}(\mathbb{F})$ generated by a $(2,3,7)$-triple whose similarity invariants are (2). Then $K$ does not fix any non-zero alternating trilinear form. 
Proof. By the above discussion we may suppose that $K$ is generated by $x=x_{6}$, $y=y_{6}$ and $z=x y$ as in (7). Proceeding by way of contradiction, let $f$ be a non-zero alternating trilinear form fixed by $K$. We have $f\left(z^{-1} v_{i}, z^{-1} v_{j}, z^{-1} v_{k}\right)=$ $\varepsilon^{i+j+k} f\left(v_{i}, v_{j}, v_{k}\right)$. Since $f$ is fixed by $z$ we get $f\left(v_{i}, v_{j}, v_{k}\right)=0$ whenever $i+j+k \not \equiv 0$ $(\bmod 7)$. Thus:

$$
f=\lambda\left(v_{1}^{*} \wedge v_{2}^{*} \wedge v_{-3}^{*}\right)+\mu\left(v_{-1}^{*} \wedge v_{-2}^{*} \wedge v_{3}^{*}\right)
$$

for some $\lambda, \mu \in \mathbb{F}$. Assume $\mu=0$. In this case $\left\langle v_{-1}, v_{-2}, v_{3}\right\rangle \leq \operatorname{rad}(f)$ and so $\operatorname{rad}(f)$ is a non-trivial subspace of $\mathbb{F}^{6}$ fixed by $K$. Since $K$ is irreducible, we obtain $\operatorname{rad}(f)=\mathbb{F}^{6}$ and so $f=0$, a contradiction. The same holds if $\lambda=0$. It follows that both $\lambda$ and $\mu$ are non-zero and, multiplying by $\lambda^{-1}$, we may assume:

$$
f=v_{1}^{*} \wedge v_{2}^{*} \wedge v_{-3}^{*}+\rho\left(v_{-1}^{*} \wedge v_{-2}^{*} \wedge v_{3}^{*}\right), \rho \neq 0
$$

i.e., $f\left(v_{1}, v_{2}, v_{-3}\right)=1, f\left(v_{-1}, v_{-2}, v_{3}\right)=\rho, f\left(v_{i}, v_{j}, v_{k}\right)=0$ otherwise. Write

$$
x=\left(a_{i, j}\right), \quad i, j \in\{1,2,-3,-1,-2,3\} .
$$

Case 1. Suppose that $A$ in (7) is diagonal. From $f\left(x v_{1}, v_{2}, v_{-3}\right)=f\left(v_{1}, x v_{2}\right.$, $\left.x v_{-3}\right)$ we get $a_{1,1}=a_{2,2} \cdot a_{-3,-3}$. Similarly, from $f\left(v_{1}, x v_{2}, v_{-3}\right)=f\left(x v_{1}, v_{2}, x v_{-3}\right)$ and $f\left(v_{1}, v_{2}, x v_{-3}\right)=f\left(x v_{1}, x v_{2}, v_{-3}\right)$ we get, respectively, $a_{2,2}=a_{1,1} \cdot a_{-3,-3}$ and $a_{-3,-3}=a_{1,1} \cdot a_{2,2}$. It follows that either $a_{1,1}=a_{2,2}=a_{-3,-3}=0$ or $a_{1,1}=a_{2,2}=$ $a_{-3,-3}=1$. In the first case $A=0$ and it is clear from (7) that $y=x(x y)$ cannot have order 3 . In the second case, $A=I$, whence the contradiction $1=\operatorname{Tr}(x(x y))=$ $\operatorname{Tr}(y)=0$.

Case 2. Some non-diagonal entry of $A$ is not zero. We may suppose that it is in the first column, up to the permutation $\left(v_{1} v_{i}\right)\left(v_{-1} v_{-i}\right)$ for some $i=2,-3$. We claim that $a_{2,1} \neq 0$ if, and only if, $a_{-3,1} \neq 0$. Indeed, suppose $a_{2,1} \neq$ 0 . The subspace $S_{1}=\left\{s \in \mathbb{F}^{6} \mid f\left(v_{1}, x v_{1}, s\right)=0\right\}$ is $x$-invariant and contains $\left\langle v_{-1}, v_{-2}, v_{3}, v_{1}\right\rangle$. If $a_{-3,1}=0$, then $v_{-3} \notin S_{1}$. As $v_{2} \in S_{1}$ the space $S_{1}$ has dimension 5 and is fixed by $K$, a contradiction. Thus $a_{-3,1} \neq 0$ and the same argument shows the opposite implication. Conjugating $x$ and $z$ by $\operatorname{diag}\left(a_{2,1}^{-1} \cdot a_{-3,1}^{-1}, a_{2,1}, a_{-3,1}, a_{2,1} \cdot a_{-3,1}, a_{2,1}^{-1}, a_{-3,1}^{-1}\right)$ their shapes are preserved as well as the condition $f\left(v_{1}, v_{2}, v_{-3}\right)=1$. Hence we may suppose $a_{2,1}=a_{-3,1}=1$ (and $a_{-1,-2}=a_{-1,3}=1$ by (7) ).

For $j \in\{-1,-2,3\}$ we obtain:

$$
0=f\left(x v_{1}, v_{1}, v_{j}\right)=f\left(v_{1}, x v_{1}, x v_{j}\right)=a_{-3, j}+a_{2, j},
$$

whence $a_{-3,-1}=a_{2,-1}, a_{-3,-2}=a_{2,-2}$ and $a_{-3,3}=a_{2,3}$. After these substitutions, for $j \in\{1,2,-3\}$ we get

$$
\rho a_{-1, j}=f\left(x v_{j}, v_{-2}, v_{3}\right)=f\left(v_{j}, x v_{-2}, x v_{3}\right)=0,
$$

whence $a_{-1,1}=a_{-1,2}=a_{-1,-3}=0\left(\right.$ and $\left.a_{-2,1}=a_{3,1}=0\right)$. Now $f\left(x v_{3}, v_{-2}, v_{j}\right)$ $=f\left(v_{3}, x v_{-2}, x v_{j}\right)$ for $j=2,-3$ gives $\rho a_{-2, j}=0$, i.e., $a_{-2,2}=a_{-2,-3}=a_{3,2}=0$. Finally $f\left(x v_{-2}, v_{3}, v_{-3}\right)=f\left(v_{-2}, x v_{3}, x v_{-3}\right)$ gives $a_{3,-3}=0$.

We conclude that $\left\langle v_{1}, v_{2}, v_{-3}\right\rangle$ is an $x$-invariant subspace, hence a $K$-invariant subspace, a contradiction.

Remark 3.2. In characteristic 2 , a perfect irreducible subgroup of $\mathrm{SL}_{6}(\mathbb{F})$ having an involution $x$ with similarity invariants $t^{2}+1, t^{2}+1, t^{2}+1$, cannot be contained in $\mathrm{SO}_{6}(\mathbb{F})^{\prime}=\Omega_{6}(\mathbb{F})$. Indeed the dimension of the fixed points space of $x$ is 3 , 
whence the quasideterminant of $x$ is, by definition, $(-1)^{3}=-1$. On the contrary, the quasideterminant of an involution in $\Omega_{6}(\mathbb{F})$ is 1 (see [4, p. xii]).

Lemma 3.3. If $H_{6}$ is irreducible, $\left\langle v_{0}\right\rangle$ has no $H_{7}$-invariant complement.

Proof. Any complement should coincide with the space $W=\left\langle v_{1}, \ldots, v_{3}\right\rangle$ generated by the eigenvectors of $\tilde{x}_{7} \tilde{y}_{7}$ relative to the eigenvalues $\neq 1$. This happens only if $a_{x}^{T}=0$ in (6). In this case $H_{6}$ preserves the restriction $\bar{Q}=Q_{\mid W}$, where $Q$ is the quadratic form fixed by $H_{7}$. The symmetric form associated to $\bar{Q}$ has Gram matrix $J_{6}$, as above, which is non-degenerate. Hence $\bar{Q}$ is a quadratic form. This implies that $H_{6}$ is contained in $\Omega_{6}(\mathbb{F})$, in contrast with Remark 3.2

Lemma 3.4. If $H_{6}$ is irreducible, then $\left\langle v_{0}\right\rangle$ is the only proper $H_{7}$-invariant subspace of $\mathbb{F}^{7}$. Similarly $W=\left\langle v_{1}, v_{2}, v_{-3}, v_{-1}, v_{-2}, v_{3}\right\rangle$ is the only proper $H_{7}^{T}$-invariant subspace of $\mathbb{F}^{7}$.

Proof. Let $U$ be a proper $H_{7}$-invariant subspace. By the irreducibility of $H_{6}$ we have either $U \cap W=0$ or $U \cap W=U$. In the first case $U$ has dimension 1 . Hence the perfect group $H_{7}$ induces the identity on $U$. This gives $U=\left\langle v_{0}\right\rangle$, the eigenspace of $\tilde{x}_{7} \tilde{y}_{7}$ relative to 1 . The second case cannot arise, since $U$ would be an $H_{7}$-invariant complement of $\left\langle v_{0}\right\rangle$, in contrast with the previous Lemma. The second part of the statement follows noting that $\left\langle v_{0}^{T}\right\rangle$ is the eigenspace of $\left(\tilde{x}_{7} \tilde{y}_{7}\right)^{T}$ relative to 1 and using the fact that $H_{6}^{T}$ is not orthogonal by Remark 3.2 .

We are now ready to prove the following result on which Theorem 1.2 is based.

Proposition 3.5. If $H_{6}$ is irreducible, then the group $H_{7}$ fixes a non-zero alternating trilinear form on $V$.

Proof. Proceeding as in the proof of Theorem 1.1. we take the action of $H_{7}$ on the space of trilinear forms, that we identify with $\Lambda^{3}\left(V^{*}\right)$, obtaining

$$
d_{\Lambda^{3}\left(V^{*}\right)}^{H_{7}}+\hat{d}_{\Lambda^{3}\left(V^{*}\right)}^{H_{7}}=d_{\Lambda^{3}\left(V^{*}\right)}^{H_{7}}+d_{\Lambda^{3}\left(V^{*}\right)}^{H^{T}} \geq 2 \text {. }
$$

It suffices to show that $d_{\Lambda^{3}\left(V^{*}\right)}^{H_{7}^{T}} \leq 1$. So let $\tilde{f}$ be a non-zero alternating trilinear form on $V$ fixed by $H_{7}^{T}$. Denote by $W$ the 6-dimensional subspace of $V$ fixed by $H_{7}^{T}$. The restriction $\left.\tilde{f}\right|_{W}$ of $\tilde{f}$ to $W$ is $H_{6}^{T}$-invariant and so it must be the zero form, by Proposition 3.1. Furthermore, the kernel of the function $\Psi: V \rightarrow \Lambda^{2}\left(V^{*}\right)$, defined as $\Psi(v)=\tilde{f}(v, *, *)$, is a $H_{7}^{T}$-invariant subspace of $V$. By Lemma 3.4 either $\operatorname{ker}(\Psi)=W$, or $\operatorname{ker}(\Psi)=0$. If $\operatorname{ker}(\Psi)=W$, then $\tilde{f}$ is the zero form. It follows that $\Psi$ is injective. Set $z=\tilde{x}_{7} \tilde{y}_{7}$ and take $\mathcal{B}$ as above. We have $z=z^{T}$ and

$$
\tilde{f}\left(z^{-1} v_{i}, z^{-1} v_{j}, z^{-1} v_{k}\right)=\varepsilon^{i+j+k} \tilde{f}\left(v_{i}, v_{j}, v_{k}\right)=\tilde{f}\left(v_{i}, v_{j}, v_{k}\right),
$$

whence either $i+j+k=0$ or $\tilde{f}\left(v_{i}, v_{j}, v_{k}\right)=0$. It follows $f\left(v_{i}, v_{j}, v_{k}\right)=0$ except, possibly, for $(i, j, k) \in\{(0, \ell,-\ell),(1,2,-3),(-1,-2,3) \mid \ell=1,2,3\}$. By the assumption that $\left.\tilde{f}\right|_{W}$ is the zero-form, we have $\tilde{f}\left(v_{ \pm 1}, v_{ \pm 2}, v_{\mp 3}\right)=0$. If $\tilde{f}\left(v_{0}, v_{i}, v_{-i}\right)=$ 0 , then $\tilde{f}\left(v_{i}, *, *\right)$ is the zero form, in contrast with the injectivity of $\Psi$. So, $\tilde{f}\left(v_{0}, v_{i}, v_{-i}\right) \neq 0$ for all $i$ 's. Substituting each $v_{i}$ with a scalar multiple, if necessary, we get $\tilde{f}=\sum_{i=1}^{3}\left(v_{0}^{*} \wedge v_{i}^{*} \wedge v_{-i}^{*}\right)$. We conclude that $d_{\Lambda^{3}\left(V^{*}\right)}^{H_{7}^{T}}=1$. 
Proof of Theorem 1.2. Suppose that char $\mathbb{F}=2$ and let $H_{6}$ be an irreducible subgroup of $\mathrm{SL}_{6}(\mathbb{F})$ generated by a non-rigid $(2,3,7)$-triple $\left(x_{6}, y_{6}, x_{6} y_{6}\right)$. Its similarity invariants are those in (2) by [20]. Setting $K=H_{6}^{T}$ in Proposition 3.1 we obtain that the transpose $H_{6}^{T}$ of $H_{6}$ does not fix non-zero alternating trilinear forms. Hence, Proposition 3.5 implies that $H_{7}=\left\langle\tilde{x}_{7}, \tilde{y}_{7}\right\rangle$, in the notation (6), fixes a nonzero alternating trilinear form $f$ on $V=\mathbb{F}^{7}$. We want to show that $f$ is similar to the Dickson form, applying [1, Theorem 5(5)]. Its hypothesis require that $H_{7}$ acts indecomposably on $V=\mathbb{F}^{7}$, which is true by Lemma 3.3. and that $\operatorname{rad}\left(B_{v_{0}}\right)=\left\langle v_{0}\right\rangle$, where $B_{v_{0}}$ is the symmetric form defined as $B_{v_{0}}(u, w)=f\left(v_{0}, u, w\right)$. It remains to show this fact. Now, the radical of $B_{v_{0}}$ is an $H_{7}$-invariant subspace of $V$. Thus, by Lemma 3.4 either $\operatorname{rad}\left(B_{v_{0}}\right)=\left\langle v_{0}\right\rangle$ or $\operatorname{rad}\left(B_{v_{0}}\right)=V$. Suppose the latter case holds, i.e. $B_{v_{0}}$ is the zero form.

Take $\bar{u}, \bar{v}, \bar{w} \in \bar{V}=V /\left\langle v_{0}\right\rangle$ and define

$$
\bar{f}(\bar{u}, \bar{v}, \bar{w})=f(u, v, w)
$$

where $u=\eta v_{0}+\bar{u}, v=\lambda v_{0}+\bar{v}$ and $w=\mu v_{0}+\bar{w}$. The function $\bar{f}$ is well-defined as

$$
f(u, v, w)=f\left(\eta v_{0}+\bar{u}, \lambda v_{0}+\bar{v}, \mu v_{0}+\bar{w}\right)=f(\bar{u}, \bar{v}, \bar{w}) .
$$

For every element of $h \in H_{7}, \bar{f}(\bar{u}, \bar{v}, \bar{w})=f(u, v, w)=f\left(h_{6}^{-1} \bar{u}, h_{6}^{-1} \bar{v}, h_{6}^{-1} \bar{w}\right)$ and so $H_{6}$ fixes a non-zero alternating trilinear form $\bar{f}$ on $\bar{V}$, in contrast with Proposition 3.1 (this time taking $K=H_{6}$ ). We conclude that $H_{7}$ fixes a Dickson form by [1, Theorem 5(5)], hence it is a subgroup of $G_{2}(\mathbb{F})$.

\section{HurWitz Generators FOR $G_{2}(q)$}

In this section we set $q=p^{a}$, where $p$ is a prime, and consider $\mathbb{F}$ as the algebraic closure of the finite field $\mathbb{F}_{q}$. Our aim is to find explicit Hurwitz generators for the groups $G_{2}(q)$ when they exist, namely for $q \geq 5[14$.

We first consider the case where $q$ is even. So let $p=2$ and $q \geq 8$. Our generators $x_{6}, y_{6}$ and their product $x_{6} y_{6}$ have similarity invariants (2), hence are conjugate to the matrices in (7), even if they have different shapes. Indeed they are obtained from family (IIa) in 25. for special values of the parameters. The choice of this family was made for uniformity with the Hurwitz generators of $\operatorname{PSp}_{6}(q), 5 \leq q$ odd, used in [22]. Indeed, it turns out that matrices of the same shape generate $\operatorname{PSp}_{6}(q)$ for $q$ odd and its subgroup $G_{2}(q)$ for $q$ even.

For each $r \in \mathbb{F}_{q} \backslash \mathbb{F}_{4}$ we set

$$
\begin{aligned}
& a=\frac{r+1}{d}, \quad b=\frac{r^{3}+r^{2}+1}{d}, \quad c=\frac{r^{3}+1}{d}, \quad d=r^{2}+r+1, \\
& x_{6}=\left(\begin{array}{cccccc}
0 & 0 & 1 & 0 & r & 1 \\
0 & 0 & 0 & 1 & 0 & a \\
1 & 0 & 0 & 0 & 1 & r \\
0 & 1 & 0 & 0 & a & 0 \\
0 & 0 & 0 & 0 & 0 & 1 \\
0 & 0 & 0 & 0 & 1 & 0
\end{array}\right), \quad y_{6}=\left(\begin{array}{cccccc}
1 & 0 & 0 & 0 & 1 & c \\
0 & 1 & 0 & 0 & b & 1 \\
0 & 0 & 0 & 0 & 1 & 0 \\
0 & 0 & 0 & 0 & 0 & 1 \\
0 & 0 & 1 & 0 & 1 & 0 \\
0 & 0 & 0 & 1 & 0 & 1
\end{array}\right)
\end{aligned}
$$

and define $H=\left\langle x_{6}, y_{6}\right\rangle$.

Lemma 4.1. The group $H$ is absolutely irreducible, except when:

$$
r^{12}+r^{9}+r^{5}+r^{2}+1=0 .
$$


In particular, if $H$ is absolutely irreducible, then it is a subgroup of $G_{2}(q)$.

Proof. For the absolute irreducibility of $H$ apply [22, Lemma 2.1] to the case $8 \leq q$ even, $r \in \mathbb{F}_{q} \backslash \mathbb{F}_{4}$. For the second claim of the statement, apply Theorem 1.2

Now we want to exclude that $H$ is contained in a maximal subgroup of $G_{2}(q)$. By [5], the absolutely irreducible maximal subgroups $M$ of $G_{2}(q)$ are of type:

$$
M \cong \mathrm{SL}_{3}(q) \cdot 2, \quad M \cong \mathrm{SU}_{3}\left(q^{2}\right) \cdot 2, \quad M \cong G_{2}\left(q_{0}\right) \quad\left(\mathbb{F}_{q_{0}}<\mathbb{F}_{q}\right) .
$$

Lemma 4.2. If $H$ is absolutely irreducible, it is not contained in $\mathrm{SL}_{3}(q)$ or $\mathrm{SU}_{3}\left(q^{2}\right)$.

Proof. If our claim is false, $H$ arises from an action of $\mathrm{SL}_{3}(\mathbb{F})$ on the symmetric square $S\left(\mathbb{F}^{6}\right)$ (see [11, 5.4.11]). But, in this action, an involution has similarity invariants $t+1, t+1, t^{2}+1, t^{2}+1$, different from those of $x_{6}$.

Remark 4.3. The field of definition of a subgroup $H$ of $\operatorname{GL}_{n}(q)$ is the smallest subfield $\mathbb{F}_{q_{1}}$ of $\mathbb{F}_{q}$ such that a conjugate of $H$, under $\mathrm{GL}_{n}(\mathbb{F})$, is contained in $\mathrm{GL}_{n}\left(q_{1}\right)\left(\mathbb{F}^{*} I\right)$, where $\mathbb{F}^{*} I$ denotes the center of $\mathrm{GL}_{n}(\mathbb{F})$. This is to ensure that no conjugate of the projective image of $H$ lies in $\operatorname{PGL}_{n}\left(q_{0}\right)$ for some $q_{0}<q_{1}$. Clearly, when $H=H^{\prime}$ is perfect, $\mathbb{F}_{q_{1}}$ coincides with the smallest subfield of $\mathbb{F}_{q}$ such that a conjugate of $H$ is contained in $\mathrm{SL}_{n}\left(q_{1}\right)$, the derived subgroup of $\mathrm{GL}_{n}\left(q_{1}\right)\left(\mathbb{F}^{*} I\right)$.

Theorem 4.4. Let $x_{6}, y_{6}$ be as in (11) with $r \in \mathbb{F}_{q} \backslash \mathbb{F}_{4}, 8 \leq q$ even, such that:

(i) $r^{12}+r^{9}+r^{5}+r^{2}+1 \neq 0$;

(ii) $\mathbb{F}_{q}=\mathbb{F}_{2}\left[r^{2}+r\right]$.

Then $H=\left\langle x_{6}, y_{6}\right\rangle=G_{2}(q)$ and there exists $r \in \mathbb{F}_{q} \backslash \mathbb{F}_{4}$ satisfying (i) and (ii).

Proof. Condition (i) implies that $H$ is absolutely irreducible (Lemma 4.1) and by the same lemma we have that $H \leq G_{2}(q)$. Let $M$ be a maximal subgroup of $G_{2}(q)$ which contains $H$. Since $H$ is a Hurwitz group, it is perfect and so it is contained in the derived subgroup $M^{\prime}$ of $M$. By [22, Lemma 3.2], the minimal field of definition of $H$ is $\mathbb{F}_{2}\left[r^{2}+r\right]$. Thus Condition (ii) gives that $M^{\prime} \neq G_{2}\left(q_{0}\right)$ for any $q_{0}$ such that $\mathbb{F}_{q_{0}}$ is a proper subfield of $\mathbb{F}_{q}$. Moreover $M^{\prime} \notin\left\{\mathrm{SL}_{3}(q), \mathrm{SU}_{3}\left(q^{2}\right)\right\}$ by Lemma 4.2. It follows that $H=G_{2}(q)$. We now prove that, for $q>4$, there exists $r \in \mathbb{F}_{q} \backslash \mathbb{F}_{4}$, where $q=2^{a}$, satisfying Conditions (i) and (ii). For each $\alpha \in \mathbb{F}_{q}$ such that $\mathbb{F}_{2}[\alpha] \neq \mathbb{F}_{q}$ there are at most two values of $r$ such that $r^{2}+r=\alpha$. Let $N(a)$ be the number of elements $r \in \mathbb{F}_{2^{a}}$ such that $\mathbb{F}_{2}\left[r^{2}+r\right] \neq \mathbb{F}_{2^{a}}$. Considering the possible subfields of $\mathbb{F}_{2^{a}}$, we get

$$
N(a) \leq 2\left(2+2^{2}+2^{3}+\ldots+2^{\left\lfloor\frac{a}{2}\right\rfloor}\right)=2^{2+\left\lfloor\frac{a}{2}\right\rfloor}-4 .
$$

Next, observe that if $a \geq 5$, then $\left(2^{2+\left\lfloor\frac{a}{2}\right\rfloor}-4\right)+12<2^{a}$. It follows that for $a \geq 5$, there exists $r \in \mathbb{F}_{2^{a}} \backslash \mathbb{F}_{4}$ satisfying Conditions (i) and (ii). Finally for $q=8,16$, it suffices to take as $r$ a generator of $\mathbb{F}_{q}^{*}$.

The following Remark gives Hurwitz generators of the Janko group $J_{2}$ over $\mathbb{F}_{4}$. Remark 4.5. For $q=4$, consider the matrices (belonging to family (IIa) of [25])

$$
x_{J_{2}}=\left(\begin{array}{cccccc}
0 & 0 & 1 & 0 & \omega & 0 \\
0 & 0 & 0 & 1 & 1 & \omega^{2} \\
1 & 0 & 0 & 0 & 0 & \omega \\
0 & 1 & 0 & 0 & \omega^{2} & 1 \\
0 & 0 & 0 & 0 & 0 & 1 \\
0 & 0 & 0 & 0 & 1 & 0
\end{array}\right), \quad y_{J_{2}}=\left(\begin{array}{cccccc}
1 & 0 & 0 & 0 & \omega^{2} & \omega^{2} \\
0 & 1 & 0 & 0 & \omega & \omega^{2} \\
0 & 0 & 0 & 0 & 1 & 0 \\
0 & 0 & 0 & 0 & 0 & 1 \\
0 & 0 & 1 & 0 & 1 & 0 \\
0 & 0 & 0 & 1 & 0 & 1
\end{array}\right),
$$


where $\omega^{2}+\omega+1=0$. By a Magma calculation the group $H=\left\langle x_{J_{2}}, y_{J_{2}}\right\rangle$ as in (12) is isomorphic to $J_{2}$.

Now, we consider the case $q \geq 5$ odd. In this case our generators have similarity invariants (3) hence are conjugate to the matrices in (6), even if they have different shapes. Indeed we take them from family (II) in 20.

For each $r \in \mathbb{F}_{q}$ define $H=\left\langle x_{7}, y_{7}\right\rangle$, where

$$
x_{7}=\left(\begin{array}{ccccccc}
0 & 0 & 0 & 1 & 0 & 0 & -4 \\
0 & 0 & 0 & 0 & 1 & 0 & r \\
0 & 0 & 0 & 0 & 0 & 1 & -3 \\
1 & 0 & 0 & 0 & 0 & 0 & -4 \\
0 & 1 & 0 & 0 & 0 & 0 & r \\
0 & 0 & 1 & 0 & 0 & 0 & -3 \\
0 & 0 & 0 & 0 & 0 & 0 & -1
\end{array}\right), y_{7}=\left(\begin{array}{ccccccc}
1 & 0 & 0 & 0 & 1 & 0 & r+2 \\
0 & 1 & 0 & 0 & 2 & 0 & 2 r+8 \\
0 & 0 & 1 & 1 & 0 & 0 & -4 \\
0 & 0 & 0 & 0 & -1 & 0 & 0 \\
0 & 0 & 0 & 1 & -1 & 0 & 0 \\
0 & 0 & 0 & 0 & 0 & 0 & -1 \\
0 & 0 & 0 & 0 & 0 & 1 & -1
\end{array}\right)
$$

Lemma 4.6. The group $H$ is absolutely irreducible, except when:

$$
d=r^{2}+15 r+100=0 .
$$

In particular, if $H$ is absolutely irreducible, then it is a subgroup of $G_{2}(q)$.

Proof. For the absolute irreducibility of $H$ see the proof of [20, Theorem 1, page 2131]. For the second claim of the statement, apply Theorem 1.1.

We notice that $\left[x_{7}, y_{7}\right]=x_{7} y_{7}^{-1} x_{7} y_{7}$ has trace 3 and characteristic polynomial (14) $\chi_{\left[x_{7}, y_{7}\right]}(t)=t^{7}-3 t^{6}-(d-5) t^{5}-(d+7) t^{4}+(d+7) t^{3}+(d-5) t^{2}+3 t-1$.

Again we want to exclude that $H$ is contained in a maximal subgroup of $G_{2}(q)$. For $q$ odd, the classification is due to P. Kleidman in [10. We summarize in Table 1 the relevant information of [3, Tables $8.41,8.42$ pages $397-398]$.

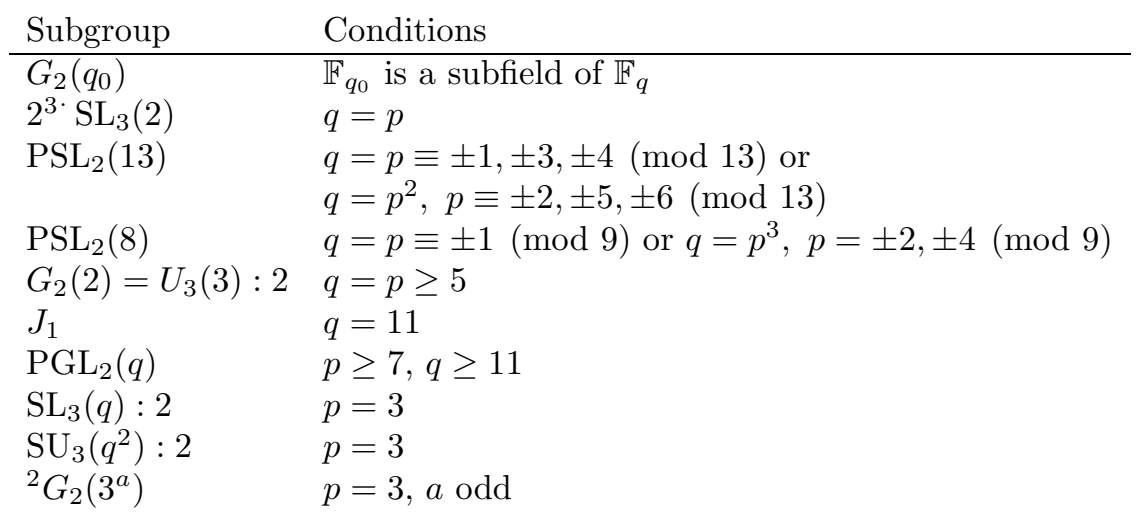

TABLE 1. Maximal irreducible subgroups of $G_{2}(q), q=p^{a}, p \neq 2$.

Since $H$ is a Hurwitz group, it is perfect. So if $H$ is contained in a maximal subgroup $M$ then $H \leq M^{\prime}$.

Lemma 4.7. Assume $5 \leq q$ odd, $H$ absolutely irreducible. Then:

(i) $H$ is never contained in any maximal subgroup $M$ isomorphic to one of the following: $\mathrm{PSL}_{2}(13), G_{2}(2), 2^{3 \cdot} \mathrm{PSL}_{3}(2), J_{1}$; 
(ii) $H$ is contained in a maximal subgroup $M \cong \mathrm{PSL}_{2}(8)$ iff $q=17$ and $r=1$.

Proof. We proceed with a case by case analysis. If $H \leq M$, then $\left(x_{7}, y_{7}, x_{7} y_{7}\right)$ must coincide with some $(2,3,7)$-triple $\left(g_{2}, g_{3}, g_{2} g_{3}\right)$ in $M$. Since the commutator $\left[x_{7}, y_{7}\right]$ has trace 3 , we are interested in those triples such that $3=\operatorname{Tr}\left(\left[g_{2}, g_{3}\right]\right)$. The possible values of these traces are read from the ordinary and modular character tables of $M$.

(a) Assume $H \leq M \cong \mathrm{PSL}_{2}$ (13), with the restrictions on $q$ given in Table 1 in particular $p \neq 3$. In this case the order of $\left[g_{2}, g_{3}\right]$ is either 6 or 7 or 13 with trace 1,0 or $(1 \pm \sqrt{13}) / 2$, respectively. Equating these values to 3 we get either $p=2$ or $p=3=q$, in contrast with our assumptions.

(b) Assume $H \leq M \cong \mathrm{PSL}_{2}(8)$ with the restrictions on $q$ given in Table 1 In this case $\left[g_{2}, g_{3}\right]$ has order 9 and its trace is a root of $t^{3}-3 t-1$. Equating its trace to 3 , we get $3^{3}-9-1=17=0$, whence $p=17=q$. On the other hand, for $q=17$, the element $\left[x_{7}, y_{7}\right]$ has order 9 only when $r=1$. In this case $H \cong \mathrm{PSL}_{2}(8)$.

(c) Assume $H \leq M \cong G_{2}(2), q=p \geq 5$. Since $H$ is perfect, we have that $H$ is actually contained in $M^{\prime}=\mathrm{SU}_{3}(3)$. In this case $\left[g_{2}, g_{3}\right]$ has order 4 . Taking $D=\left[x_{7}, y_{7}\right]^{4}$, we have $D_{7,7}=5 r-7$. If $p=5$, then $D_{7,7}=3 \neq 1$. If $p \neq 5$, set $r=\frac{8}{5}$. Then $D_{2,7}=-\frac{36}{5} \neq 0$, since $p \geq 7$.

(d) Assume $H \leq M \cong 2^{3 \cdot} \mathrm{PSL}_{3}(2)$ with $q=p \geq 5$. If $p \neq 7$, then the trace of $\left[g_{2}, g_{3}\right]$ is in $\{0, \pm 1\}$. As in (a) above we get either $p=2$ or $p=3=q$, against our assumptions. If $q=p=7$, then $\left[x_{7}, y_{7}\right]$ has order $\geq 19$, so it is cannot be an element of $2^{3 \cdot} \mathrm{PSL}_{3}(2)$.

(e) Assume $M \cong J_{1}$ with $q=11$. The element $\left[g_{2}, g_{3}\right]$ has order $10,11,15$ or 19 with respective traces $9,7,5$ and 4 . Since $\operatorname{Tr}\left(\left[x_{7}, y_{7}\right]\right)=3$ we have a contradiction.

Lemma 4.8. If $p=3$ and $H$ is absolutely irreducible, then it is not contained in $\mathrm{SL}_{3}(q)$ or $\mathrm{SU}_{3}\left(q^{2}\right)$.

Proof. The Hurwitz subgroups of $\mathrm{SL}_{3}\left(q^{2}\right)$ are isomorphic to $\mathrm{PSL}_{2}(7)$ or to $\mathrm{PSL}_{2}(27)$. However $\mathrm{PSL}_{2}(27)$ does not have irreducible representations of degree 7 . If $H$ is contained in $\mathrm{PSL}_{2}(7)$, then $\left[x_{7}, y_{7}\right]$ has order 4 , whence $\operatorname{Tr}\left(\left[x_{7}, y_{7}\right]\right)=-1$, a contradiction.

Lemma 4.9. Assume $H$ absolutely irreducible. Then two triples $\left(x_{7}\left(r_{1}\right), y_{7}\left(r_{1}\right)\right.$, $\left.z_{7}\left(r_{1}\right)\right)$ and $\left(x_{7}\left(r_{2}\right), y_{7}\left(r_{2}\right), z_{7}\left(r_{2}\right)\right)$ are conjugate if, and only if, $r_{1}=r_{2}$.

Proof. Assume that $\left(x_{7}\left(r_{1}\right), y_{7}\left(r_{1}\right), z_{7}\left(r_{1}\right)\right)$ and $\left(x_{7}\left(r_{2}\right), y_{7}\left(r_{2}\right), z_{7}\left(r_{2}\right)\right)$ are conjugate. Then the characteristic polynomial of $\left[x_{7}\left(r_{1}\right), y_{7}\left(r_{1}\right)\right]$ and $\left[x_{7}\left(r_{2}\right), y_{7}\left(r_{2}\right)\right]$ are the same, whence $r_{1}^{2}+15 r_{1}=r_{2}^{2}+15 r_{2}$. Suppose that $r_{1} \neq r_{2}$. Then $r_{1}=-15-r_{2}$. Now, consider the element $w=\left[x_{7}, y_{7}\right]^{2} y_{7}\left(x_{7} y_{7}\right)^{2}$ whose characteristic polynomial is

$$
\chi_{w}(t)=t^{7}+f_{1}(r) t^{6}+f_{2}(r) t^{5}-f_{3}(r) t^{4}+f_{3}(r) t^{3}-f_{2}(r) t^{2}-f_{1}(r) t-1
$$

where $f_{1}(r)=r^{3}+25 r^{2}+250 r+999$. From $f_{1}\left(r_{1}\right)=f_{1}\left(r_{2}\right)$ we get $d\left(2 r_{2}+15\right)=0$. Since $H$ is absolutely irreducible, then $d \neq 0$ and so $r_{2}=-\frac{15}{2}$. However, in this case $r_{1}=-15-r_{2}=-\frac{15}{2}=r_{2}$, a contradiction. We conclude that $r_{1}=r_{2}$. 
As mentioned in the Introduction, the group $\operatorname{PSL}_{2}(q)$ is Hurwitz when either $q=p \equiv 0, \pm 1(\bmod 7)$ or $q=p^{3}$ and $p \equiv \pm 2, \pm 3(\bmod 7)$. Moreover, in $\operatorname{Aut}\left(\mathrm{PSL}_{2}(q)\right)=\mathrm{P}_{2}(q)$, there are 3 conjugacy classes of Hurwitz triples for $q=p \equiv \pm 1(\bmod 7)$, only one otherwise.

Theorem 4.10. Let $q=p^{a}, p$ odd, $q \geq 5$, and let $x_{7}, y_{7}$ be as in (13) with $r \in \mathbb{F}_{q}$ such that $r \neq 1$ if $q=17$ and $r^{2}+15 r+100 \neq 0$. Assume further that $\mathbb{F}_{q}=\mathbb{F}_{p}[r]$. Then one of the following holds:

(i) $H=G_{2}(q)$;

(ii) $H=\operatorname{PSL}(2, q)$ with $q=p$ if $p \equiv 0, \pm 1(\bmod 7), q=p^{3}$ if $p \equiv \pm 2, \pm 3$ $(\bmod 7)$.

Moreover, in case (ii) it is always possible to choose $r$ such that $H=G_{2}(q)$.

Proof. The group $H$ is absolutely irreducible by Lemma4.6 and the assumption $r^{2}+$ $15 r+100 \neq 0$ and so it is a subgroup of $G_{2}(q)$. Suppose that there exists a maximal subgroup $M$ of $G_{2}(q)$ containing $H$. Condition $\mathbb{F}_{q}=\mathbb{F}_{p}[r]$ implies that $\mathbb{F}_{q}$ is the field of definition of $H$ (see [20, Remark 6]). Thus $M \neq G_{2}\left(q_{0}\right)$ for every $q_{0}<q$. By Lemma 4.7 we may also exclude $M^{\prime} \in\left\{\mathrm{PSL}_{2}(13), \mathrm{PSL}_{2}(8), G_{2}(2), 2^{3 \cdot} \mathrm{SL}_{3}(2)\right\}$. When $q=3^{a}$, we may also exclude $M^{\prime} \in\left\{\mathrm{SL}_{3}(q), \mathrm{SU}_{3}\left(q^{2}\right)\right\}$ by Lemma 4.8 If $a$ is odd, suppose $M \cong{ }^{2} G_{2}\left(3^{a}\right)$. By [2, Proposition 3.14] two semisimple elements of ${ }^{2} G_{2}\left(3^{a}\right)$ which have the same trace are conjugate. Now $x_{7} y_{7}$ has trace 0 and, for $p=3$, also $\left[x_{7}, y_{7}\right]$ has trace 0 . So $\left[x_{7}, y_{7}\right]^{7}$ must be a 3 -element and so has trace 1 . However this gives $r\left(r^{2}-1\right)=0$ and so $r \in \mathbb{F}_{3}$, a contradiction with the assumption $\mathbb{F}_{q}=\mathbb{F}_{3}[r]$. Thus $H$ is not contained in any subgroup of Table 1 , except possibly in $\mathrm{PGL}_{2}(q)$. We conclude that either $H=G_{2}(q)$ or $H \leq M^{\prime}$ with $M \cong \mathrm{PGL}_{2}(q)$. Assume that the latter case holds. By the classification of the Hurwitz subgroups of $\mathrm{PSL}_{2}(q)$ 13 we have $H \cong \operatorname{PSL}_{2}(q)$ with $q=p$ if $p \equiv 0, \pm 1(\bmod 7), q=p^{3}$ if $p \equiv \pm 2, \pm 3(\bmod 7)$.

In $G_{2}(q)$ there is just one conjugacy class of maximal subgroups isomorphic to $\mathrm{PGL}_{2}(q)$. Thus, by the information given before the statement, there are at most 3 non-conjugate Hurwitz triples in $G_{2}(q)$ which can generate $\mathrm{PSL}_{2}(q)$. By Lemma 4.9. different values of $r$ give rise to non conjugate triples. So we have to exclude at most 3 values of $r$ to avoid $H \leq \mathrm{PGL}_{2}(q)$. Clearly we have to exclude at most other 2 values of $r$ for the absolute irreducibility. We also note that, if $H \leq \mathrm{PGL}_{2}(q)$, then $q \neq 17$ since $17 \equiv 3(\bmod 7)$. Our last claim now follows from the inequalities: $p \geq 7>2+3$ if $q=p \equiv 0, \pm 1(\bmod 7)$, and $p^{3}-p>2+3$ if $q=p^{3}$.

For sake of completeness, we provide Hurwitz generators also for the Janko group $J_{1}$. Consider the following matrices $x_{J_{1}}, y_{J_{1}} \in \mathrm{SL}_{7}(11)$ (Family (I) of [20, with $r_{3}=2$ and $r_{4}=4$ ):

$$
x_{J_{1}}=\left(\begin{array}{ccccccc}
0 & 0 & 0 & 1 & 0 & 0 & -1 \\
0 & 0 & 0 & 0 & 1 & 0 & 5 \\
0 & 0 & 0 & 0 & 0 & 1 & 2 \\
1 & 0 & 0 & 0 & 0 & 0 & -1 \\
0 & 1 & 0 & 0 & 0 & 0 & 5 \\
0 & 0 & 1 & 0 & 0 & 0 & 2 \\
0 & 0 & 0 & 0 & 0 & 0 & -1
\end{array}\right), \quad y_{J_{1}}=\left(\begin{array}{ccccccc}
1 & 0 & 0 & 0 & 4 & 0 & 3 \\
0 & 1 & 0 & 0 & 8 & 0 & -1 \\
0 & 0 & 1 & 1 & 0 & 0 & 9 \\
0 & 0 & 0 & 0 & -1 & 0 & 0 \\
0 & 0 & 0 & 1 & -1 & 0 & 0 \\
0 & 0 & 0 & 0 & 0 & 0 & -1 \\
0 & 0 & 0 & 0 & 0 & 1 & -1
\end{array}\right) .
$$

Again, by a Magma calculation, the group $H=\left\langle x_{J_{1}}, y_{J_{1}}\right\rangle$ is isomorphic to $J_{1}$. 


\section{REFERENCES}

[1] M. Aschbacher, Chevalley Groups of Type $G_{2}$ as the Group of a Trilinear Form, J. Algebra 109, 193-259 (1987).

[2] H. Bäärnhielm, Recognising the small Ree groups in their natural representations, J. Algebra 416 (2014), 139-166.

[3] J.N. Bray, D.F. Holt and C.M. Roney-Dougal, The maximal subgroups of the low-dimensional finite classical groups, London Mathematical Society Lecture Note Series, 407. Cambridge University Press, Cambridge, 2013

[4] J.H. Conway, R.T Curtis, S.P. Norton, R.A. Parker and R.A. Wilson, Atlas of finite groups. Maximal subgroups and ordinary characters for simple groups, Oxford University Press, Eynsham, 1985.

[5] B.N. Cooperstein, Maximal Subgroups of $G_{2}\left(2^{n}\right)$, J. Algebra 70, 23-36 (1981).

[6] J. Dieudonné, Sur les groupes classiques, Actualités scientifiques et industrielles 1040, Herman, Paris, 1948

[7] L. Di Martino, M.C. Tamburini and A. Zalesskii, On Hurwitz groups of low rank, Comm. Algebra 28 (2000) no. 11, 5383-5404.

[8] L. Finkelstein and A. Rudvalis, Maximal subgroups of the Hall-Janko-Wales group, J. Algebra 24 (1973), 486-493.

[9] N. Jacobson, Basic algebra I, W.H. Freeman and Co., San Francisco, Calif., 1974.

[10] P.B. Kleidman, The maximal subgroups of the Chevalley groups $G_{2}(q)$ with $q$ odd, the Ree groups ${ }^{2} G_{2}(q)$, and their automorphism groups, J. Algebra 117 (1988), no. 1, 30-71.

[11] P. Kleidman and M. Liebeck, The subgroup structure of the finite classical groups, London Mathematical Society Lecture Note Series, 129. Cambridge University Press, Cambridge, 1990.

[12] S. Lang, Linear algebra. Third edition, Undergraduate Texts in Mathematics. SpringerVerlag, New York, 1987.

[13] A.M. Macbeath, Generators of the linear fractional groups, Proc. Symp. Pure Math. 12 (1969), 14-32.

[14] G. Malle, Hurwitz groups and $G_{2}(q)$, Canad. Math. Bull. 33 (1990), no. 3, 349-357.

[15] C. Marion, On triangle generation of finite groups of Lie type, J. Group Theory, 13 (2010), 619-648.

[16] C.-H. Sah, Groups related to compact Riemann surfaces, Acta Math. 123 (1969) 13-42.

[17] L.L. Scott, Matrices and cohomology, Ann. Math. 105 (1977), 473-492.

[18] M.C. Tamburini and M. Vsemirnov, Hurwitz groups and Hurwitz generation. Handbook of algebra. Vol. 4, 385-426, Elsevier/North-Holland, Amsterdam, 2006.

[19] M.C. Tamburini and M. Vsemirnov, Irreducible (2,3,7)-subgroups of $\operatorname{PGL}_{n}(F), n \leq 7, J$. Algebra 300 (2006), 339-362.

[20] M.C. Tamburini and M. Vsemirnov, Irreducible $(2,3,7)$-subgroups of $\operatorname{PGL}_{n}(F), n \leq 7$, II., J. Algebra 321 (2009), no. 8, 2119-2138.

[21] M.C. Tamburini and A. Zalesskii, Classical groups in dimension 5 which are Hurwitz, Proceedings of the Gainesville Conference on Finite Groups, 2003, Editors: C.Y. Ho, P. Sin, P.H. Tiep, A. Turull, Walter de Gruyter, (2004), 363-371.

[22] M.C. Tamburini Bellani and M. Vsemirnov, Hurwitz generation of $\operatorname{PSp}_{6}(q)$, Comm. Algebra 43 (2015), no. 10, 4159-4169.

[23] K.B. Tchakerian, An explicit $(2,3,7)$-generation of the simple Ree groups ${ }^{2} G_{2}\left(3^{n}\right), C . R$. Acad. Bulgare Sci. 58 (2005), no. 7, 749-752.

[24] R. Vincent and A.E. Zalesski, Non-Hurwitz classical groups, LMS J. Comp. Math. 10 (2007), $21-82$.

[25] M. Vsemirnov and M.C. Tamburini, Irreducible $(2,3,7)$-subgroups of $\mathrm{PGL}_{n}(F), n \leq 7$, III (in preparation).

Dipartimento di Matematica e Fisica, Università Cattolica del Sacro Cuore, Via Musei 41, I-25121 Brescia, ItAly

E-mail address: marcoantonio.pellegrini@unicatt.it

Dipartimento di Matematica e Fisica, Università Cattolica del Sacro Cuore, Via Musei 41, I-25121 Brescia, ItAly

E-mail address: mariaclara.tamburini@unicatt.it 